\title{
Pelvic Incidence (PI) is more Easily Understood as the Pelvic Base Angle (PBA)
}

Keywords: Incidence; Base-angle; Pelvis; Compensation

Received: March 03, 2016; Accepted: April 14, 2017; Published: April 18, 2017

\section{Commentary}

Evolution of the human clade to obtain an erect posture and bipedal locomotion has been made possible through a series of skeletal morphological changes. The pelvic vertebra has played a crucial role in this transformation by undergoing progressive widening and retroversion [1]. Maintenance of an upright posture requires the body's center of gravity to fall over a narrow area between the feet while maintaining a horizontal gaze with minimal energy expenditure [2]. In 1992, Duval-Beaupere et al. studied the pelvic shape and sagittal profile of healthy volunteers using a Barycentermeter [3]. They described a morphological parameter called the "Pelvic Incidence" to enable reproducible analysis of the anatomical characteristics of the pelvis in the sagittal plane. The pelvic incidence (PI) is measured as an angle formed by two vectors: 1 ) The line joining the bicoxo-femoral axis to the center of the sacral end plate and 2) A line perpendicular to the sacral endplate. Morphologically, the line joining the bicoxofemoral axis and the center of sacrum represents the pelvic thickness in the sagittal plane (SPT) $[4,5]$. This vector represents the line of transmission of the body weight from the pelvis to the lower limbs and anatomically, it passes through the strongest parts of the pelvis: postero-superior acetabular wall and the sciatic buttress. Measurement of this vector alone provides little information as it fails to take into consideration, the orientation of the sacrum on which, the entire weight of the spine rests. Since, the sacrum is a curvilinear bone; the best vector describing its direction in the vertical axis is a line perpendicular to the sacral end-plate. The pelvic incidence thus determines the relative position of the sacral plate with respect to the femoral heads. It decides upon on the amount of lumbar lordosis required to maintain an erect posture [6]. In degenerative changes of the lumbar spine, as the lumbar spine flattens, a mis-match is created between the required and the existing values of lumbar lordosis. This causes the gravity line to pass forward of the bicoxo-femoral axis and in an uncompensated patient, this leads to a forward stooped posture causing pain and disability [7-9].

The pelvic incidence is a fixed morphological parameter, whose value remains more or less constant throughout adult life [10]. It is an algebraic sum of two dynamic angles: the pelvic tilt and the

\section{Subaraman Ramchandran, Aaron Buckland and Thomas J Errico}

\author{
NYULMC Hospital for Joint Diseases, New \\ York, USA
}

\begin{abstract}
Corresponding author:
Subaraman Ramchandran
\end{abstract}

ほ subbu_gsmc@yahoo.in

MBBS, MS (Orth) NYULMC Hospital for Joint Diseases, New York, USA.

Citation: Ramchandran S, Buckland A, Errico TJ. Pelvic Incidence (PI) is more Easily Understood as the Pelvic Base Angle (PBA). Spine Res. 2017, 3:1.

sacral slope [3]. The pelvic tilt represents the inclination of the inominate bones with respect to the frontal plane and the sacral slope determines the sacral tilt with respect to the horizontal plane. This can be explained by the fact that, verticalization of the sacrum and horizontalization of the pelvic ring occurs as pelvic tilt increases. The result of verticalization of the sacrum is decrease in the sacral slope. Although in theory the term "pelvic incidence" is aptly given to this three-dimensional morphological parameter and may be clear to engineers, it is confusing to clinicians who are making measurements on a two-dimensional X-ray. Clinically pelvic incidence can be visualized by the sagittal profile of the waist-line and buttocks (Figure 1).

The use of the term "incidence" in science is miscellaneous. In physics, incidence is defined as an angle made by a vector to a normal drawn to the surface of its contact. In aerodynamics, angle of incidence is defined as the angle made by a line connecting the leading and trailing edges of an airfoil with the horizontal. In geometry, incidence refers to a binary relation between different types of object in space (For example; relationship between a point and a line, a line and a plane and so on). Also, the term incidence is generally referenced with respect to an element/ object in space. For example, angle of incidence of a light ray is referenced to the surface of reflection. Usually, this plane of reference is understood and does not need to be quoted. For pelvic incidence, this surface or plane of reference is the frontal plane passing through the bicoxo-femoral axes. Therefore, the term pelvic incidence creates confusion since clinicians are 
imaging the relationship between the femoral heads, sacral endplate and sacrum in a lateral two-dimensional plane. The lines drawn on the $\mathrm{X}$-ray represent a simple angle represented by the vertex in the middle of the sacral endplate and two rays one to the center of the femoral heads and the second perpendicular to the endplate. In two-dimensional space this angle is best described as the "Pelvic Base Angle". This is because the entire pelvis can be regarded as a "Pelvic Vertebra" forming the base of the spinal column (Jean Dubousset, 1972). Pelvic Incidence is best described in a three-dimensional space as an angle formed at the intersection of two planes which represent the vertical axes of the two major bones forming the pelvic ring namely: the inominate and the sacrum (Figure 2). The entire pelvis can be regarded as the Pelvic vertebra which forms the base of the spinal column, the angle describing its orientation in two-dimensional space can be best termed as "Pelvic Base angle".

For further simplification, let us consider the pelvis as a vertical rectangular cube. The way this rectangle is laid out describes PBA or PI. The three factors that decide the pelvic incidence of an individual are: posterior sacral offset- horizontal distance between femoral head center and center of sacral endplate, vertical pelvic offset- shortest vertical distance between femoral head center and center of sacral endplate and the orientation of the sacro-iliac joint, or the obliquity with which the sacrum articulates with the ilium through the sacroiliac joints. Thus it can be seen that when the pelvic overhang increases, vertical pelvic offset decreases or the sacro-iliac joint inclination increases, pelvic incidence increases (Figure 3).

This discussion is in no means an attempt to alter the ground breaking description of PI by Duval-Beaupere. There is no doubt that the lack of clinical understanding of the important concepts has hindered and slowed mental adaptation. Hence, it would be

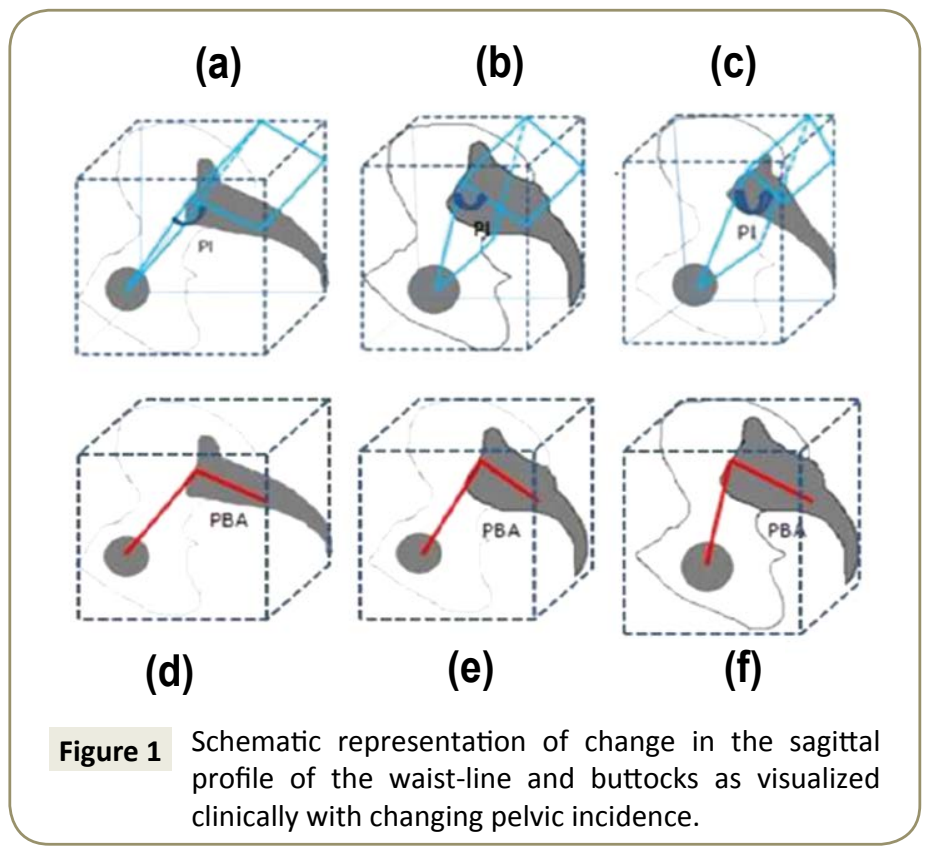

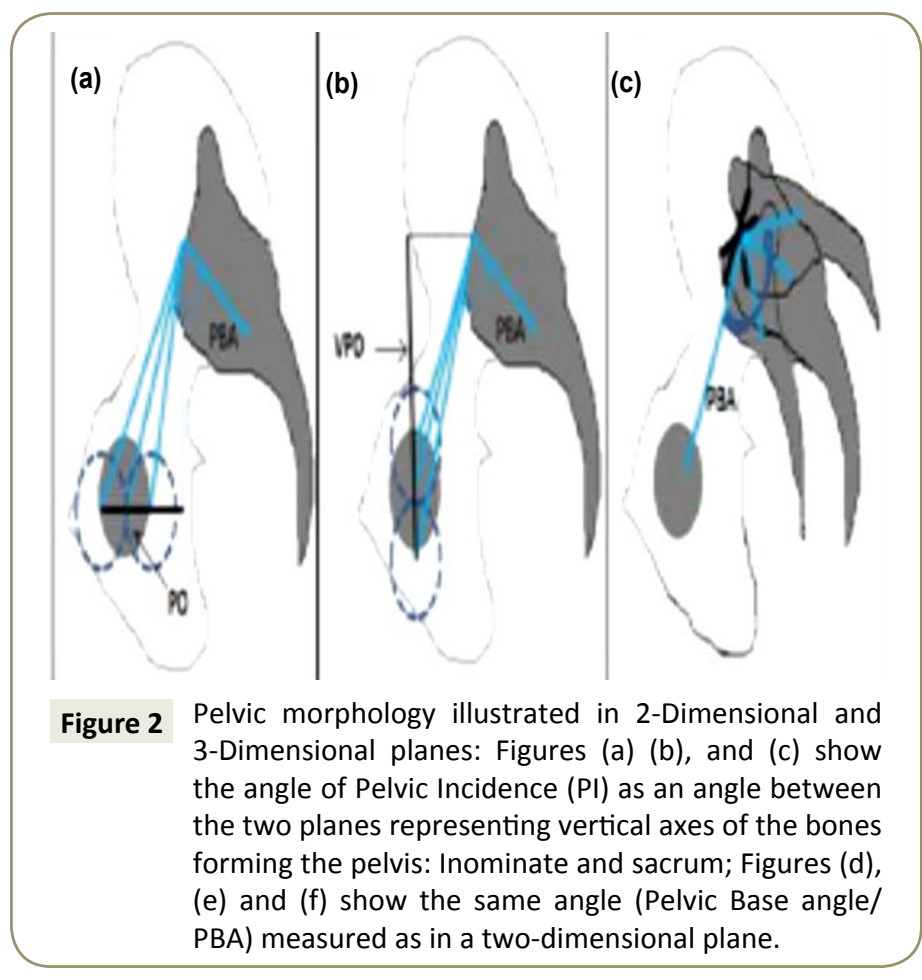

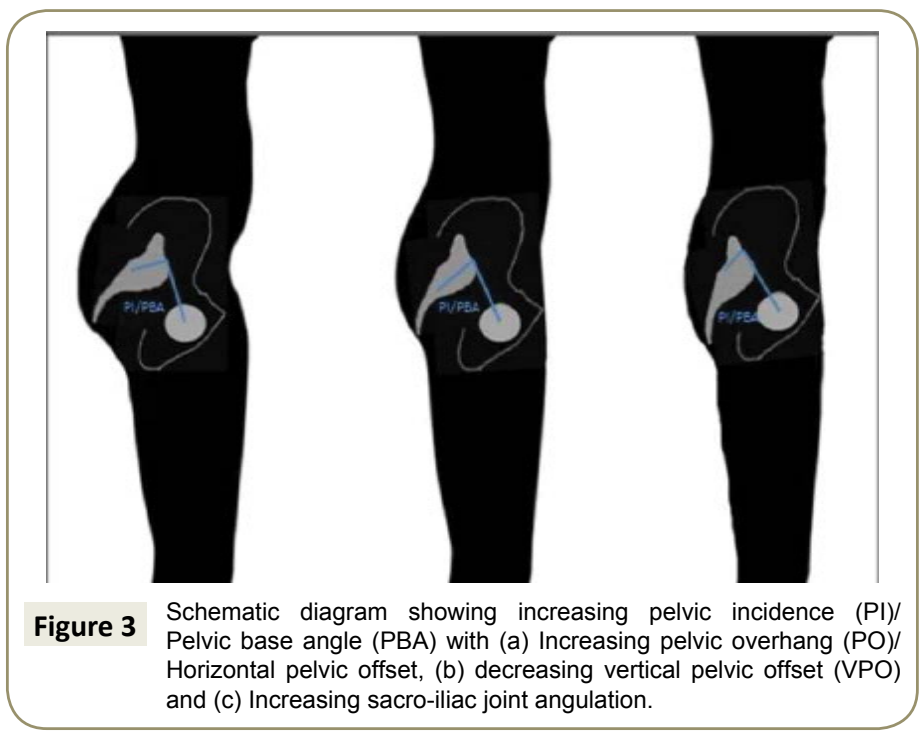

logical to describe pelvic incidence when measured on a twodimensional X-ray as the "pelvic base angle" or PBA.

Nonetheless, the pelvic incidence is an extremely important parameter determining the global spinal balance of an individual. Surgeons should maintain a low threshold in accessing the pelvic morphology in patients with regional spinal pathologies. The term "pelvic base angle" is useful descriptive terminology in explaining simply the concept of pelvic incidence to a broader audience including patients, medical trainees and even health professionals. 


\section{References}

1 Schmitt D (2003) Insights into the evolution of human bipedalism from experimental studies of humans and other primates. J Exp Biol 206: $1437-1448$.

2 Dubousset J (1994) Three-dimensional analysis of the scoliotic deformity. Pediatr Spine 479-496.

3 Duval-Beaupère $G$, Schmidt $C$, Cosson $P$ (1992) A barycentremetric study of the sagittal shape of spine and pelvis: The conditions required for an economic standing position. Ann Biomed Eng 20: 451-462.

4 Le Huec JC, Aunoble S, Philippe L, Nicolas P (2011) Pelvic parameters: origin and significance. Eur Spine J 20(5): 564-571.

5 Legaye J, Duval-Beaupère G, Hecquet J, Marty C (1998) Pelvic incidence: A fundamental pelvic parameter for three-dimensional regulation of spinal sagittal curves. Eur Spine J 7: 99-103.
6 Sparrey CJ, Bailey JF, Safaee M, Clark AJ, Lafage V, et al. (2014) Etiology of lumbar lordosis and its pathophysiology: a review of the evolution of lumbar lordosis, and the mechanics and biology of lumbar degeneration. Neurosurg. Focus 36: E1.

7 Terran J, Schwab F, Shaffrey Cl, Smith JS, Devos P, et al. (2013) The SRS-schwab adult spinal deformity classification: Assessment and clinical correlations based on a prospective operative and nonoperative cohort. Neurosurgery 73: 559-568.

8 Yamada K, Abe Y, Yanagibashi Y, Hyakumachi T, Satoh S (2015) Midand long-term clinical outcomes of corrective fusion surgery which did not achieve sufficient pelvic incidence minus lumbar lordosis value for adult spinal deformity. Scoliosis 10: S17.

9 Ailon T, Smith JS, Shaffrey Cl, Lenke LG, Brodke D, et al. (2015) Degenerative spinal deformity. Neurosurgery 77: S75-S91.

10 Vialle R, Levassor N, Rillardon L, Templier A, Skalli W, et al. (2005) Radiographic analysis of the sagittal alignment and balance of the spine in asymptomatic subjects. J Bone Joint Surg Am 87: 260-267. 